\title{
Application of Parallel Computing to Obtain all Complex Roots of a High Order Equation
}

\author{
Liying Wang ${ }^{\mathrm{a}}$, Zhanlin $\mathrm{Yu}^{\mathrm{b}}$ \\ School of Information Science and Technology, Bohai University, Jinzhou, 121013, China \\ a986558686@qq.com, ${ }^{b} 284189433 @ q q . c o m$
}

Keywords: a high order equation; complex roots; genetic algorithm; parallel computing

\begin{abstract}
Solving the high order equation to get the complex roots is of great importance for analysis and synthesis of the engineering projects. A few methods, such as the Optimal Trinomial Factor method and the Splitting Trinomial Factor method, can be used to solve these equations. However, these methods may not converge. In addition, as they have computing residuals optimizing a trinomial factor of the algebra equation, it has an influence on the accuracy of the calculation. To solve the shortcomings of the traditional method, a parallel method for solving the complex roots of a high order equation is proposed, which has combined the genetic algorithm and parallel computing. This method uses its strong global convergence and better optimization ability. In determining the appropriate initial population and scale, the method can be used to obtain all the complex roots quickly and efficiently. The results of numerical experiments show the validity and convergence of the proposed method, and obtain the full number of roots of the equation to ensure the accuracy of the calculation results.
\end{abstract}

\section{Introduction}

In the field of numerical calculation, the solution of a high order equation such as:

$$
\mathrm{F}(x)=\sum_{i=0}^{n} a_{i} x^{i}=0
$$

It is often solved by the Newton iteration method with fast convergence speed [1,2,3]. The basic idea is to determine the initial value of $x_{k}$, and then in accordance with the iterative formula to calculate. The form of the iterative formula is the following representation,

$$
x=x_{k}-\frac{f\left(x_{k}\right)}{f_{\left(x_{k}\right)}^{\prime}}(k=0,1, \cdots)
$$

The calculation is stopped when an approximate real number root satisfying the accuracy is obtained. However, the Newton iteration method can only obtain the real solutions. In the field of engineering project analysis and engineering computing, complex roots have a very important role. For example, in the process of fault diagnosis, to extract comprehensive rotor fault feature, the full spectrum and the complex local mean decomposition are combined to obtain the complex signal of the rotor under certain conditions, and the complex signal is divided into series of complex product functions followed in descending order by energy [4]. For the complex wavelet bivariate filtering model, the bivariate Bayesian estimation algorithm is extended to the complex field, which can change the influence of the increase of the signal phase noise to the filtering effect, and has a strong ability to suppress the interference of the interferogram. The edge and details of the information [5]. In the study of the viscous factors to consider the issue of wave propagation, due to the introduction of viscous and naturally get the imaginary units of the dispersion equation. The diffusion equations obtained in these problems are also included in the imaginary units [6].

So far, the method of solving the complex roots of a high order equation is relatively few. The commonly used method is the splitting factor method $[7,8]$. The basic idea is as follows. Any real coefficient algebraic equation can only be a pair of conjugate root. Therefore, the polynomial in the algebraic equation of the high-order real coefficient can be decomposed into a number of quadratic 
factors and a factor of one factor (only the highest power of the equation is odd). Finding a quadratic factor is equivalent to finding a pair of conjugate roots or two real roots. The cleavage factor method can avoid the complex operation, but the splitting factor method is slow to converge in the process of solving the problem, and it may not converge.

The paper study the high order equations which only have complex roots and no real solutions. First, it shows the processes and methods of simplifying Problem (1). Second, in the case of avoiding complex arithmetic, the genetic algorithm and the parallel computation are combined to solve problems. Then the paper is to report five numerical results. In the case where the number of roots is not lost, the approximate root of the guarantee is close to the real root.

\section{Preliminaries}

We consider the formula of the higher order equation

$$
f(x)=\sum_{j=0}^{n} \alpha_{j} x^{j}=0 \quad(0 \equiv n \bmod 2)
$$

$x=a+b i$ is another way of expressing to the complex roots. Equation (3) can be changed into

$$
f(a+b i)=\sum_{j=0}^{n} \alpha_{j}(a+b i)^{j}=0
$$

The Equation (4) is expanded according to the binomial theorem. The binomial theorem is as follows.

$$
(a+b)^{n}=\sum_{r=0}^{n} C_{n}^{r} a^{n-r} b^{r}
$$

and $C_{n}^{r}=\frac{n !}{r !(n-r) !}$. Then the equation (4) is expanded and reduced by using the binomial theorem, which can be transformed into a binary higher order system, which is in the following form

$$
\left\{\begin{array}{l}
y_{1}=\sum_{j=0 k=0}^{2 n} \sum^{\frac{j}{2}} \frac{j !}{(j-2 k) !(2 k) !}(-1)^{k} a^{j-2 k} b^{2 k}=0, \\
y_{2}=\sum_{j=0 k=0}^{2 n} \sum^{\frac{j+1}{2}} \frac{j !}{(j-2 k-1) !(2 j+1) !}(-1)^{k} a^{j-2 k-1} b^{2 k} i=0
\end{array}\right.
$$

We denote $c=b^{2}$ and $r=\sqrt{a^{2}+c^{2}}$, then $\left\{\begin{array}{l}a=r \cos \alpha \\ c=r \sin \alpha\end{array}, \alpha \in(0, \pi)\right.$. The binary higher order equations are transformed into

$$
\left\{\begin{array}{l}
y_{1}=\sum_{j=0 k=0}^{2 n} \sum^{\frac{j}{2}} \frac{j !}{(j-2 k) !(2 k) !}(-1)^{k} r^{j-k}(\sin \alpha)^{k}(\cos \alpha)^{j-2 k}=0 \\
y_{2}=\sum_{j=0 k=0}^{2 n} \sum^{\frac{j+1}{2}} \frac{j !}{(j-2 k-1) !(2 j+1) !}(-1)^{k} r^{j-k-1}(\sin \alpha)^{k}(\cos \alpha)^{j-2 k-1} i=0
\end{array}\right.
$$

\section{The Approach}

Parallel computing is one of the most effective way to achieve high performance computing $[9,10]$. Traditional serial computing is affected by physical speed, and then its application is limited. For complex scientific engineering problems, in order to improve the accuracy of the calculation, the process of solving the problem will involve a large amount of calculation. Compared with the calculation process of serial computing, parallel computing uses multiple threads to solve the same problem, that is, the problem of complex computational complexity is decomposed into several sub 
problems. Through the rational distribution of threads, the calculation of each sub problem is completed. The results of the synthesis of sub-problems to complete the calculation of the whole problem. In view of the need for high-precision calculation and faster calculation of speed, this paper uses parallel computing.

Genetic algorithm is a kind of evolutionary search algorithm which simulates the evolutionary process of natural biological evolution process. It has the characteristics of simplicity, robustness and versatility. In the genetic algorithm, each solution in the search space is represented as a chromosome, and a potential solution of the problem is constructed in the form of chromosomes into the initial population of the algorithm. The population will evolve and the chromosomes with poor fitness values in the population will be removed by the fitness function. The chromosomes with better fitness values will be passed to the next generation until the chromosomes whose fitness values meet the accuracy requirements are found in the population. The optimal solution of the problem. Genetic algorithms are widely used to solve complex optimization problems. As an NP-hard problem, job shop scheduling problem (JSSP) is an important research topic in combinatorial optimization and production management. Genetic algorithm is used to find the optimal solution for JSSP. The efficiency of search is increased and the convergence is improved in JSSP with the genetic algorithm [11]. Using the good convergence performance of genetic algorithm to deal with the problem of optimization to ensure that the new individual has the characteristics of only migrating to the cloud once, reducing the computing time of the optimal solution, satisfying the application execution time requirement while minimizing the energy consumption of the mobile terminal [12].

The basic steps of this paper are as follows. First, the initial generation is randomly generated. Then, the genetic algorithm will use the three genetic operators of selection, crossover and mutation to generate the next generation population. After generating new populations, each chromosome in the population was evaluated using the fitness function and the chromosomes with higher fitness values were retained. If the fitness value of the chromosome satisfies the termination condition, the algorithm ends and the optimal solution is found. Otherwise, the above process is repeated until the chromosome satisfying the requirement is found. In the process of evaluating the chromosomes by the fitness function, the speed of the new population is accelerated by judging the chromosomal fitness value by the multi-thread assignment. The solution of the complex root of Problem (1) is reduced to the solution of the binary high order equation for $r$ and $\alpha$ in Equation (7) and Equation (8). The fitness value is set to $\min =0.01 . y=\left(r_{1}-r_{2}\right)^{2}$ is the fitness function. The initial population generation interval is $[0, \pi]$. Equation (7) and Equation (8) determine the value of min corresponding to $\alpha$. The algorithm process is shown in Fig. 1.

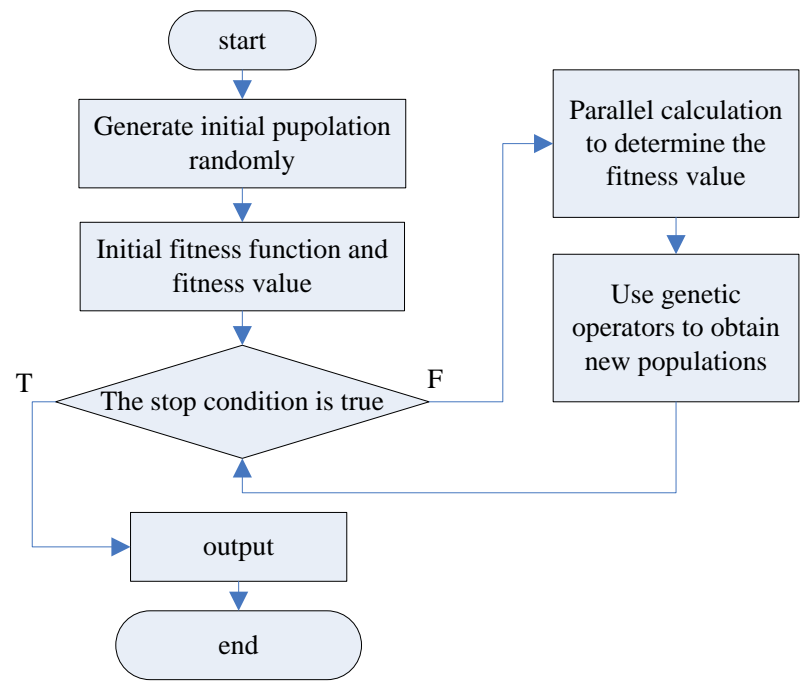

Fig. 1. The algorithm process 


\section{Numerical Results}

The purpose of this paper is to quickly solve the complex root of a higher order equation. In the case of ensuring that the solution of the exact high-precision equation is obtained, there is no case of missing solution. The equation for the experiment is 12th order. The numerical results of this paper are shown in table 1 and a brief analysis is made.

Table.1. Numerical results

\begin{tabular}{|c|c|}
\hline coefficient & $1,-1.5,-9,7.2,3,0,-304,3,0,0,2,-109,38798000$ \\
\hline$r$ & $4.604077641,8.934910275,15.707603819$, \\
& $15.883751597,8.935764917,4.344055167$ \\
\hline coefficient & $0.206167553,1.197696875,1.490212798$, \\
& $1.636715823,1.914357664,2.896079704$ \\
\hline$r$ & $-37.289,56.001,-10.908,5.319,9.2,-7.5$, \\
& $-1.909,14.067,-7.09,0,18.07,8.901,-121023$ \\
\hline$\alpha$ & $2.083812679,2.367754653,3.535032778$, \\
& $3.543892371,2.273223912,1.811446933$ \\
\hline coefficient & $0.111989522,0.870912576,1.393040608$, \\
& $1.682839483,2.168598402,3.001505937$ \\
\hline$r$ & $-1.98,13,-9,-107.2,-0.903,1$, \\
& $4.79,-13,-0.982,25,-90.8,9,-20110$ \\
\hline$\alpha$ & $1.758639922,6.554279228,2.521605674$, \\
& $2.9317594377,1.9786580667,2.094583861$ \\
\hline coefficient & $0.245429687,0.835278125,1.227148004$, \\
& $1.665921875,2.277587566,3.112048437$ \\
\hline$r$ & $109,-0.392,-940.87,-10.56,-190.03,1.309$, \\
& $-84.51,0.13,2,25,-9.118,61.036,908845748728$ \\
\hline$\alpha$ & $7.179580691,22.284817867,40.791916781$, \\
& $40.806330756,22.283008349,7.200287711$ \\
\hline coefficient & $0.412321932,1.353076088,1.527651321$ \\
& $1.613921871,1.788572801,2.757178905$ \\
\hline$r$ & $-1.98,13,-9,-107.2,-0.903,1$, \\
& $4.79,-13,-0.982,25,-90.8,9,-20110$ \\
\hline$\alpha$ & $2.347214904,3.166095112,5.045394598$, \\
& $5.004092635,3.071667371,2.282313602$ \\
\hline & $0.157075189,1.001355125,1.440126511$, \\
& $1.684556256,2.130329682,2.986425158$ \\
\hline
\end{tabular}

Experiments were carried out with a number of complex roots, the image openings toward different unity of the higher order equations. The coefficients of the equation are arranged from high to low order in table 1. Values of $r$ and $\alpha$ according to the above formula can be converted to $a$ and $b$. Then we can show target complex roots of the problem. The experimental results show that the genetic algorithm combined with parallel computing method to solve a high degree univariate polynomial equation of all the complex roots of the effectiveness and feasibility. The test of the population size of the same equation shows that the error between the values of the solution is not obvious, but the calculation time will increase with the size of the population increased significantly. This shows that the impact of population size on the algorithm is mainly reflected in the time, that is, the increase in the size of the population will increase the cost of time, but will not have a greater impact on the performance of the algorithm. In addition, the experimental results show that the value of $r$ is affected by the change of $\alpha$. The bigger the $r$, the greater the effect on $\alpha$. For $a$ and $b$ in the Equation (6) and Equation (7), the smaller the value of the two variables, the higher the accuracy of $r$ and $\alpha$.

\section{Conclusion}

Solving all the complex roots of a high order equation can play a great help to the theory and application of elastic wave theory, fault diagnosis and so on. The splitting factor method can solve the complex root of the equation, but its convergence is uncertain and its application is limited. We 
use genetic algorithm combined with parallel computing method to solve a high degree univariate polynomial equation in this paper. The genetic algorithm is used to guarantee the global convergence of the algorithm. The use of parallel computing can significantly improve the speed of computing advantages. In this paper, we find the full number of roots of the equation, which avoids the complex operation and has high precision. The experimental results show that the method is correct and effective. As the genetic algorithm is simple and easy to implement, and this paper combines it with the parallel computing, the computing speed can basically meet the needs of practical applications. Because of the random distribution of the complex roots of the high order equation, it is very important to determine the initial population, and the influence of factors such as coefficients and exponents is considered for specific problems.

\section{References}

[1] Wang Yuan, Li Qiu-hong, Huang Xiang-hua, "Numerical calculation of aero-engine model based on self-tuning Broyden quasi-Newton method," Journal of Aerospace Power, vol. 31, no. 1, pp. 249-256, 2016.

[2] Wang Yi, Dong Liang-Guo, "Multi-Parameter full waveform inversion for acoustic VTI media using the truncated Newton method," Chinese Journal of Geophysics, vol. 58, no. 8, pp. 2873-2885, 2015.

[3] C.Liu, X.T.Tian, Y.H.Cao, etc "Selected harmonic elimination in cascaded multi-level voltage inverters based on the stochastic Newton method (SNM)," Power System Protection and Control, vol. 45, no. 5, pp. 96-102, 2017.

[4] C.J.Hu, Y.J.Meng, W.p.Lei, J.Han "Application of Decomposition Full - Vector Envelope Technique in Complex Local Part and Its Application in Rotor Fault Feature Extraction," Journal of Mechanical Engineering, vol. 52, no. 7, pp. 69-78, 2016.

[5] Y.H.Hw, J. J. Zhu, etc, "An Bivariate Filtering Algorithm for Interfere Complex Wavelet Complex Domain," Acta Geodaetica et Cartographica Sinica, vol. 45, no. 5, pp. 574-580, 2016.

[6] Yuan L, Du J, Ma T, et al, "Study on SH-SAW in imperfectly bonded piezoelectric structures loaded with viscous liquid," Acta Mechanica, vol. 225, no. 1, pp. 1-11, 2014.

[7] Philippe Chartier, Florian Me, hats, Mechthild Thalhammer, Yong Zhang, "Improved error estimates for splitting methods applied to highly-oscillatory nonlinear Schrodinger equations," Mathematics of Computation, pp. 2863-2885, 2016.

[8] Chuanqing Gu, Lei Wang. "On the multi-splitting iteration method for computing PageRank," Journal of Applied Mathematics and Computing, vol. 42, no. 1, pp. 479-490, 2013.

[9] D.X.Xie, D.C.Mei, P.Ding, "Natural gas dynamic simulation algorithm based on parallel computing," Journal of Computer Applications, vol. 36, pp. 44-48, 2016.

[10] Y.Q Zhu, W. Luo, "A data mining parallel algorithm based on dynamic scheduling," Modern Electronics Technique, vol. 39, no. 15, pp. 156-163, 2016.

[11] T.F.Zhang, Y.Ma, etc, "Improved Genetic Algorithm for Flexible Job Shop Scheduling Problem," Journal of Chinese Computer System, vol. 38, no. 1, pp. 129-132, 2017.

[12] X.L, J.B. Li, Z.Yang, Z.J.Li, "A Task Collaborative Execution Policy in Mobile Cloud Computing," Chinese Journal of Computers, vol. 40, no. 2, pp. 364-377, 2017. 